Received: 2015.01 .28
Accepted: 2015.02 .10
Published: 2015.01 .05
Authors' Contribution:
A Study Design
B Data Collection
C Statistical Analysis
D Data Interpretation
E Manuscript Preparation
F Literature Search
G Funds Collection

\section{Neuroendocrine Tumor of the Gallbladder}

\author{
Ahmed Monier ${ }^{1 \mathrm{ABCDEF}}$, Nehal Saloum ${ }^{1 \text { BCF }}$, Wojciech Szmigielski ${ }^{2 \mathrm{CDEF}}$, \\ Amal Alrashid ${ }^{10 E F}$, Sarbar M.E. Napaki ${ }^{3 \mathrm{CDEF}}$
}

1 Department of Clinical Imaging, Hamad General Hospital, Hamad Medical Corporation, Doha, Qatar
2 Department of Clinical Imaging, National Center for Cancer Care and Research, Hamad Medical Corporation, Doha, Qatar
${ }^{3}$ Department of Laboratory Medicine and Pathology, Hamad General Hospital, Hamad Medical Corporation, Doha, Qatar

Author's address: Wojciech Szmigielski, Department of Clinical Imaging, National Center for Cancer Care and Research, Hamad Medical Corporation, P.0. Box 3050, Doha, Qatar, e-mail:w.szmigielski@gmail.com and wszmigielski@hmc.org.qa

\section{Summary}

Background: The primary neuroendocrine tumors of the gallbladder are very rare, representing $0.2 \%$ of all neuroendocrine tumors. The definite diagnosis is incidental in most cases.

Case Report: A 60-year-old male patient presented with a one-month history of epigastric pain and jaundice, itching, flushing, cough and wheezing. Ultrasound demonstrated the gallbladder containing multiple stones, with wall thickening and an adjacent liver lesion. Additionally, those structures showed noticeable contrast enhancement in CT and MRI. Histopathological examination revealed a primary gallbladder neuroendocrine tumor.

Conclusions: Though a rare entity, primary neuroendocrine tumor should be considered in the differential diagnosis of gallbladder cancers.

MeSH Keywords:

Diagnosis • Gallbladder Neoplasms • Magnetic Resonance Imaging • Multidetector Computed Tomography • Neuroendocrine Tumors • Ultrasonography

PDF file: http://www.polradiol.com/abstract/index/idArt/893705

\section{Background}

Neuroendocrine tumors (NET) of the gallbladder (GB) are uncommon and represent only $0.2 \%$ of all NETs [15]. Carcinoid syndrome is very infrequent $(<1 \%)$, and most GB carcinoids are incidentally diagnosed during a histological examination of GB samples at autopsy, after cholecystectomy for acute or chronic cholecystitis, or after surgery for other suspected biliary pathology [1-6]. NETs of GB probably originate from either a multipotent stem cell or neuroendocrine cells in intestinal or gastric metaplasia of the gallbladder epithelium, which occurs following cholelithiasis and/ or chronic inflammation [7]. The presentation of our case of NET of GB is justified by its rarity and its typical clinical presentation along with nonspecific imaging findings, emphasizing the need for further evaluation and specific histopathological analysis of any suspicious GB carcinoma.

\section{Case Report}

A 60-year-old male patient presented with epigastric pain and jaundice, flushing, cough and wheezing.
Ultrasound examination demonstrated distended GB, containing multiple calculi (with the largest one measuring $18 \mathrm{~mm}$ ) and focal thickening of the gallbladder wall in the fundus, measuring about $7 \mathrm{~mm}$. An irregular in outline, heterogeneous lesion of mixed echogenicity was found in the right hepatic lobe, adjacently to the GB fossa (Figure 1). There was also mild intrahepatic biliary tree dilatation.

Contrast-enhanced CT and MRI displayed a distended GB with thickened and enhancing wall, more at the fundus. The liver showed an irregular, multiloculated, hypodense but marginally enhancing focal lesion surrounding the GB bed (Figures 2 and 3). The lesion had a necrotic center and it was inseparable from the thickened GB wall. Dilatation of the intrahepatic biliary radicles and the proximal segment of the common bile duct (CBD) was noted, likely due to compression of the distal CBD segment caused by presence of a large porto-caval necrotizing lymph node. The radiological diagnosis suggested GB carcinoma with direct liver invasion and secondary porto-caval lymphadenopathy, causing biliary obstruction. 


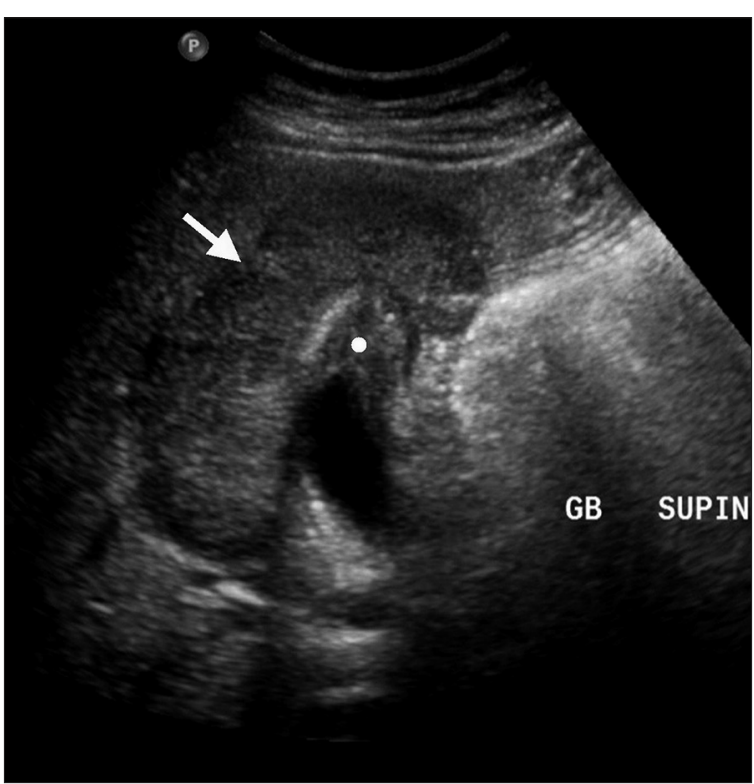

Figure 1. Ultrasound of GB: Well defined, irregular heterogeneous mixed hypo- and iso-echoic mass at the gallbladder fossa (arrow) burst the overlying liver parenchyma and with direct invasion of gallbladder wall at fundus (white dot), giving the possibilities of either of hepatic or gallbladder origin.

The staging of our case was T3N1M0, according to the American Joint Committee for TNM classification of gallbladder cancer [8].

A core biopsy was taken from the gallbladder mass. Histopathological examination showed cores of a malignant tumor, characterized by irregular nests and trabeculae of epithelial cells within a fibrous stroma. The cells showed a moderate amount of eosinophilic cytoplasm and a low to focally moderate degree of nuclear pleomorphism. On immunohistochemistry, the tumor was strongly and diffusely positive for neuroendocrine markers Chromogranin A (Figure 4), Synaptophysin and CD56 (Figure 5). The tumor was also focally positive for Cytokeratin-7 and polyclonal CEA. Focal nuclear staining for CDX2 was also noted. The tumor was negative for S-100, CK20, CD10, Cytokeratin-19, prostate-specific antigen, TTF-1, HepParl, and RCC. There was a high proliferation index, approximately $60 \%$ (Figure 6).

The appearances were those of a neuroendocrine carcinoma consistent with GB origin.

\section{Discussion}

Carcinoid tumors are quite unusual endocrine tumors arising mainly in the gastrointestinal tract, where they involve less than $2 \%$ of all primary gastrointestinal tumors [1]. Primary carcinoid tumors originate frequently in the appendix, jejunum, and rectum. Less common sites include the bronchial epithelium, duodenum, colon, and stomach. In particular, GB is exceptionally infrequent site for carcinoid [9]. Sanders described only 7 tumors $(0.2 \%)$ in GB among 3633 digestive tract carcinoids [10]. Godwin described also only one case $(0.04 \%)$ in $\mathrm{GB}$, from among

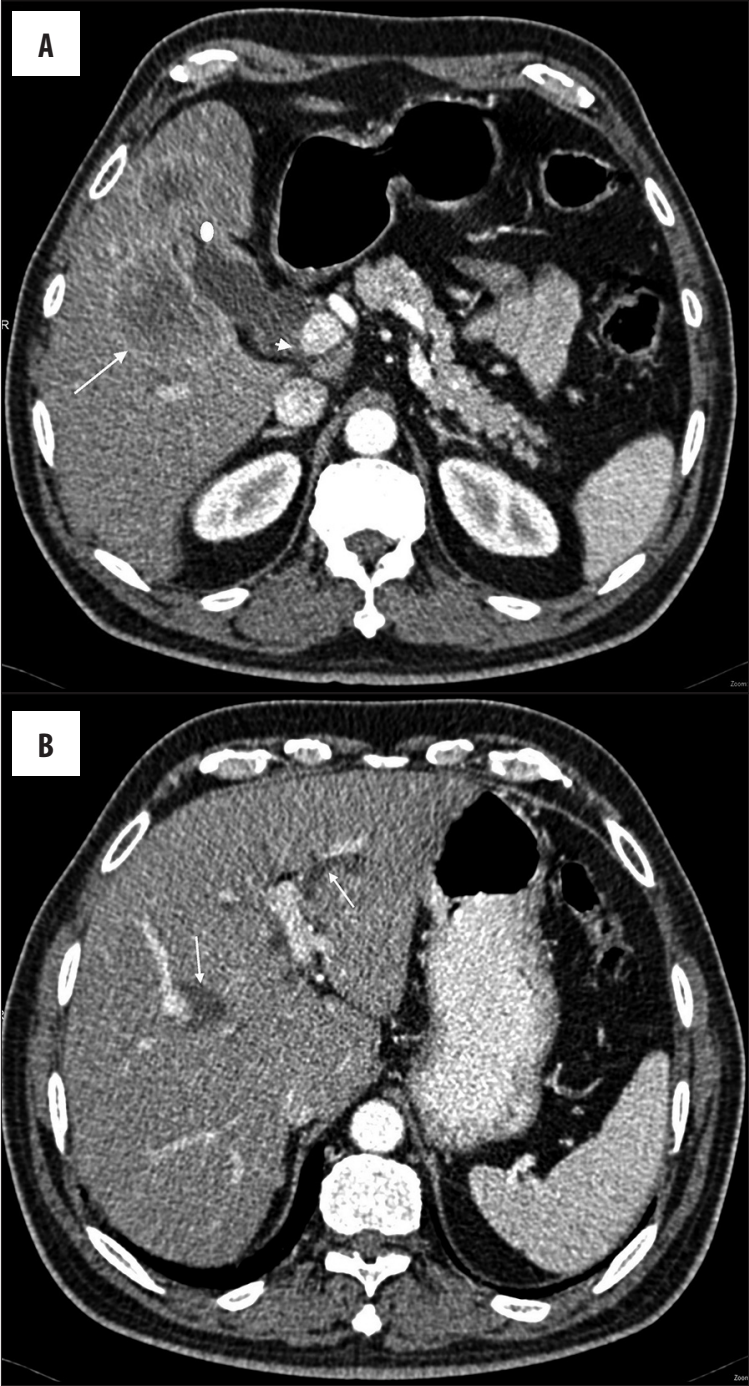

Figure 2. Contrast enhanced CT scan of the abdomen: (A) Enhancing focal wall thickening at GB fundus (white dot), inseparable from multi-loculated liver lesion (arrow), which shows strong peripheral enhancement with central necrosis, GB stones (arrow head). (B) Dilated intrahepatic biliary ducts (arrows).

2837 carcinoids [11]. It is difficult to diagnose GB carcinoid tumor before surgery. The diagnosis is infrequently made by radiological imaging, because most patients have nonspecific manifestations and radiological findings are similar to presentations of any other GB tumors [9]. The age at presentation of GB NETs ranges from 38 to 81 years, and there is a markedly higher incidence in women [12]. Most of the GB carcinoids were detected incidentally during a routine histological examination of GB samples after surgical removal, when cholecystectomy for cholecystitis or treatment of patients with suspected biliary malignancy is performed [13-22]. MRI is the modality of choice in staging GB tumors. When combined with MRCP it will give a full overview of the biliary ducts in case of highly suspected biliary duct invasions in patients who presented with jaundice. Reported sensitivity rates for direct hepatic invasion and lymph node invasion on MRI can be as high as 100\% and $92 \%$, respectively [7]. 


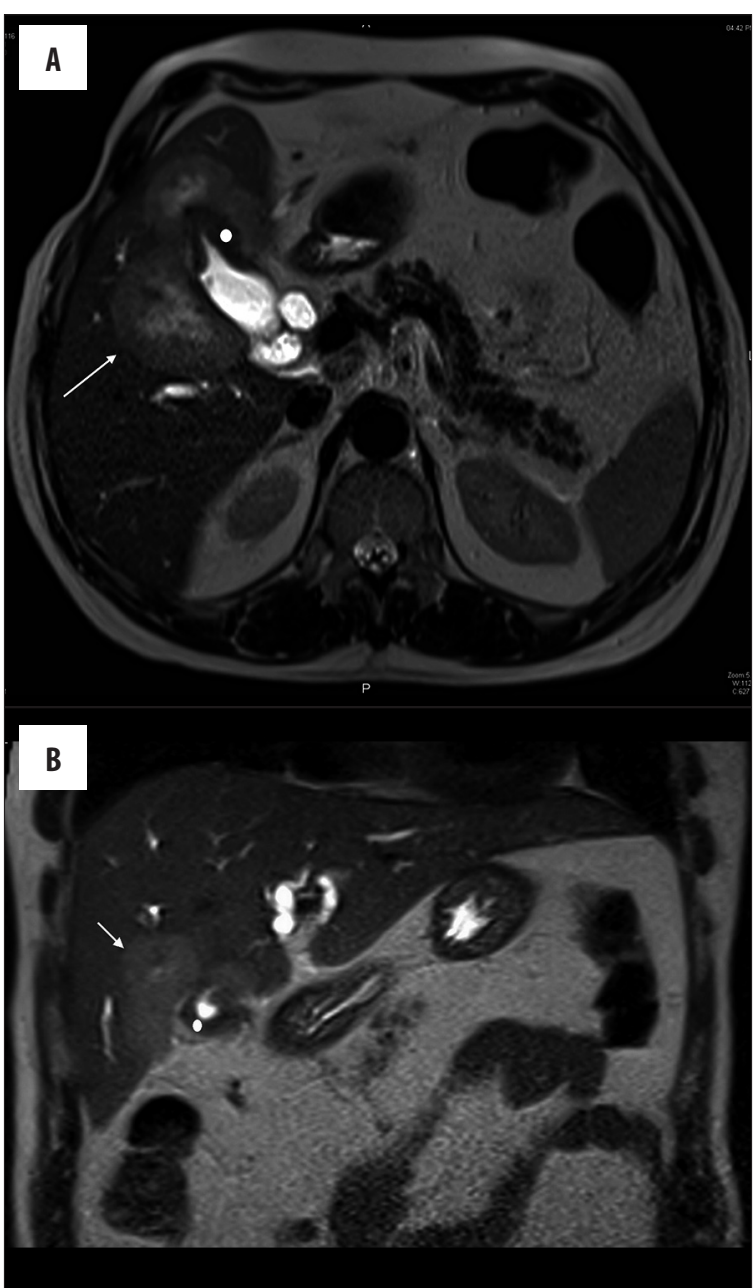

Figure 3. T2 axial (A) and coronal (B) MRI of abdomen: Marked wall thickening of $\mathrm{GB}$ fundus, impressive of primary gallbladder neoplasm (white dot). Multiple matted liver lesions centered on GB fundus seen moderately hyperintense, impressive of direct hepatic invasion (arrow).

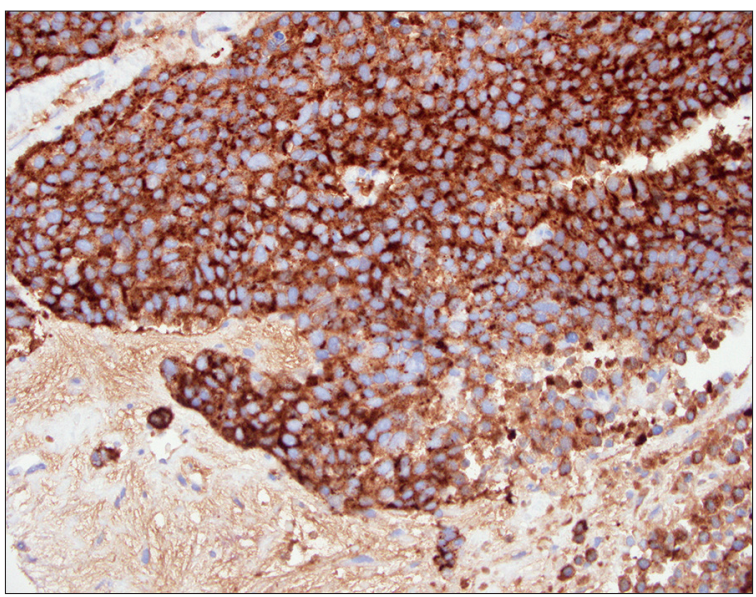

Figure 4. Microscopic examination of GB biopsy specimen: diffuse cytoplasmic staining for chrmogranin $A ; \times 400$ magnification immunoperoxidase stain.

When possible, surgical treatment, with the goal of whole resection, is the gold standard for typical carcinoids of GB.

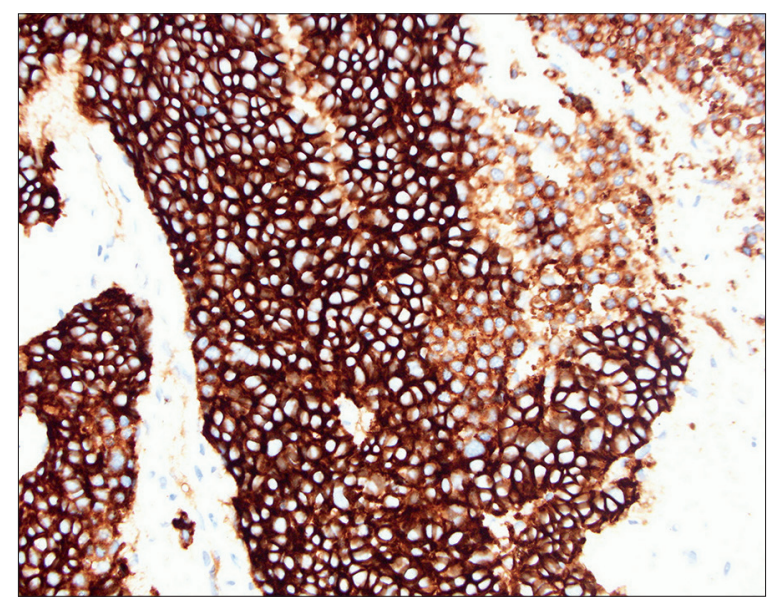

Figure 5. Microscopic examination of GB biopsy specimen: diffuse cytoplasmic staining for $\mathrm{CD} 56 ; \times 400$ magnification immunoperoxidase stain.

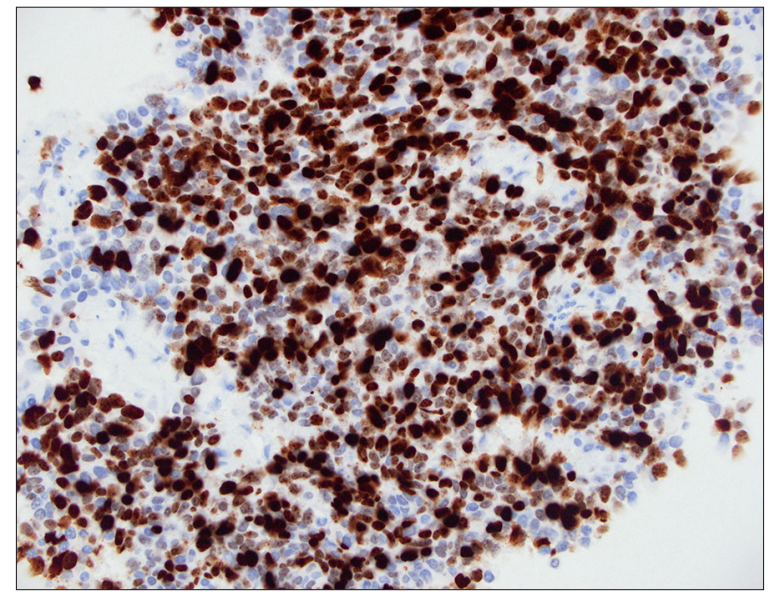

Figure 6. Microscopic examination of GB biopsy specimen: : High proliferation index (nuclear staining for MIB-1); $\times 400$ magnification immunoperoxidase stain.

For pre-invasive and early-detected cancers (Tls and Tl), simple cholecystectomy is possibly a suitable therapy. For advanced lesions, a more aggressive radical surgery, including radical cholecystectomy and regional lymphadenectomy combined with a hepatic resection in order to obtain adequate free margins, is needed [8]. For several years, sieric $\mathrm{CgA}$ and urinary 5-HIAA, each of which has a specificity of nearly 100\% but a low sensitivity, have been the gold standard for discovering carcinoids and conducting follow-ups [23]. The new introduction of SPECT/CT and PET/CT hybrid systems results in fusion images by which the anatomical location of the functional findings may be perfectly determined. PET with Fludeoxyglucose (18F) is an established technique for oncological imaging but has not proven useful for NETs, except for highly aggressive tumors [24,25]. Based on the ability of NETs to take up decarboxylate amine precursors, 11C-labeled and 18F-labeled amine precursors such as 5-HTP and L-DOPA have been used to visualize NETs $[26,27]$. In carcinoid tumors, 18F-L-DOPA-PET performs better than SRS, with the reverse situation for non-carcinoid NETs [28]. The 11C-5-HTP-PET is superior to CT and SRS with the maximum profits being the provision of complementary diagnostic information regarding small 
tumors, detection of possible recurrences, and visualization of occult tumors [28]. The $68 \mathrm{Ga}$ is a generator-produced positron emitter and does not need an in-house cyclotron. Current data indicate that 68Ga-DOTA-TOC-PET is effective in the identification of metastatic NETs. However, at this time, 68Ga generators are not widely available [10].

\section{Conclusions}

We are of the opinion that NET of GB, though a rare entity, should be considered in the differential diagnosis of GB carcinoma.

\section{References:}

1. Deehan DJ, Heys SD, Kernohan N, Eremin O: Carcinoid tumor of the gallbladder. Gut, 1993; 34: 1274-76

2. Nishigami T, Yamada M, Nakasho $\mathrm{K}$ et al: Carcinoid tumor of the gall bladder. Intern Med, 1996, 35: 953-56

3. Kaiho T, Tanaka T, Tsuchiya S et al: A case of classical carcinoid tumor of the gallbladder: review of the Japanese published works. Hepatogastroenterology, 1999; 46: 2189-95

4. Khetan N, Bose NC, Arya SV, Gupta HO: Carcinoid tumor of the gallbladder: report of a case. Surg Today, 1995; 25: 1047-49

5. Konishi E, Nakashima Y, Smyrk TC, Masuda S: Clear cell carcinoid tumor of the gallbladder. A case without von Hippel-Lindau disease. Arch Pathol Lab Med, 2003; 127: 745-47

6. Porter JM, Kalloo AN, Abernathy EC, Yeo CJ: Carcinoid tumor of the gallbladder: laparoscopic resection and review of the literature. Surgery, 1992; 112: 100-5

7. Gallbladder. In: Edge SB, Byrd DR, Compton CC et al. (eds.): AJCC Cancer Staging Manual. $7^{\text {th }}$ ed. New York, NY: Springer, 2010; 211-17

8. Eltawil KM, Gustafsson BL, Kidd M, Modlin IM: Neuroendocrine tumors of the gallbladder: An evaluation and reassessment of management strategy. J Clin Gastroenterol, 2010; 44(10): 687-95

9. Zou YP, Li WM, Liu HR, Li N: A case report of primary carcinoid tumour of the gallbladder. World J Surg Oncol, 2010; 8: 12

10. Sanders RJ, Springfield, Charles C. Thomas: Carcinoid of the Gastrointestinal Tract, 1973, 10

11. Godwin DJ: Carcinoid tumors, an analysis of 2937 cases. Cancer 1975, 36: 560-69

12. Schwartz LH, Black J, Fong Y et al: Gallbladder carcinoma: findings at MR imaging with MR cholangiopancreatography. J Comput Assist Tomogr, 2002; 26(3): 405-10

13. Mizukami Y, Nagashima T, Ikuta K et al: Advanced endocrine cell carcinoma of the gallbladder. Hepatogastroenterology, 1998; 45: 1462-67

14. Yokoyama Y, Fujioka S, Kato K et al: Primary Carcinoid tumor of the gallbladder: Resection of a case metastasizing to the liver and analysis of outcomes. Hepatogastroenterology, 2000; 47(31): 135-39

15. Ozawa K, Kinoshita M: A case of double carcinoid tumors of the gallbladder. Dig Dis Sci, 2003; 48(9): 1760-61
16. Modlin JM, Shapiro MD, Kidd M: An analysis of rare carcinoid tumors: clarifying these clinical conundrums. World J Surg, 2005 29: 92-101

17. Anjaneyulu V, Shankar-Swarnalatha G, Rao SC: Carcinoid tumor of the gall bladder. Ann Diagn Pathol, 2007; 11: 113-16

18. Geo SK, Harikumar R, Kumar S et al: Gall bladder carcinoid: a case report and review of literature. Trop Gastroenterol, 2007; 28(2): 72-73

19. Modlin IM, Lyes KD, Kidd M: A 5-decade analysis of 13,715 carcinoid tumors. Cancer, 2003; 97: 934-59

20. Soga J: Primary endocrinomas (carcinoids and variant neoplasms) of the gallbladder. A statistical evaluation of 138 reported cases. J Exp Clin Cancer Res, 2003; 22: 5-15

21. Z'graggen K, Birrer S, Maurer CA et al: Incidence of port site recurrence after laparoscopic cholecystectomy for preoperatively unsuspected gallbladder carcinoma. Surgery, 1998; 124: 831-38

22. Modlin IM, Kidd M, Drozdov I et al: Pharmacotherapy of neuroendocrine cancer. Expert Opin Pharmacother, 2008; 9(15): 2617-26

23. Bajetta E, Ferrari L, Martinetti A et al: Chromogranin a, neuron specific enolase, carcinoembryonic antigen, and hydroxyindole acetic acid evaluation in patients with neuroendocrine tumors. Cancer, 1999; 86: 858-65

24. Adams S, Baum R, Rink T et al: Limited value of fluorine-18 Fluorodeoxyglucose positron emission tomography for the imaging of neuroendocrine tumours. Eur J Nucl Med, 1998; 25: 79-83

25. Pasquali C, Rubello D, Sperti C et al: Neuroendocrine tumor imaging: can 18F-fluorodeoxyglucose positron emission tomography detect tumors with poor prognosis and aggressive behavior. World J Surg, 1998; 22: 588-92

26. Koopmans KP, de Vries EG, Kema IP et al: Staging of carcinoid tumours with 18F-DOPA PET: a prospective, diagnostic accuracy study. Lancet Oncol, 2006; 7: 728-34

27. Montravers F, Grahek D, Kerrou K et al: Can fluorodihydroxyphenylalanine PET replace somatostatin receptor scintigraphy in patients with digestive endocrine tumors. J Nucl Med, 2006; 47: 1455-62

28. Koukouraki S, Strauss LG, Georgoulias V: Evaluation of the pharmacokinetics of 68Ga-DOTATOC in patients with metastatic neuroendocrine tumors scheduled for 90Y-DOTATOC therapy. Eur J Nucl Med Mol Imaging, 2006; 33(4): 460-66 